\title{
Imprinted Polymers and Grephene-MIP composite based Chemical Sensors for the Selective Detection of Heavy Metal lons
}

\author{
Ghulam Mustafar ${ }^{1,2}$, Maimoona Yasinzai ${ }^{1}$, Peter A. Lieberzeit ${ }^{2}$ \\ ${ }^{1}$ Sulaiman Bin Abdullah Aba-Al-Khail Center for Interdisciplinary Research in Basic Sciences, \\ International Islamic University $\mathrm{H}-10$ Islamabad, Pakistan \\ ${ }^{2}$ Department of Physical Chemistry, University of Vienna, Waehringer Strasse A-1090 Vienna, Austria \\ gmustafa@iiu.edu.pk
}

Key words: Molecular imprinted polymers, composite, heavy metals, sensitivity, selectivity

\begin{abstract}
The fabrication of highly sensitive, selective, rapid and convenient sensors is of substantial interest for the real-time and online environmental monitoring, aliment safety, clinical diagnoses ${ }^{1,2}$. Numerous conventional techniques are in practice for the detection and analysis of heavy metals in water samples however, these techniques are unable to be applicable for real-time and online monitoring ${ }^{2-4}$. It is of substantial interest to develop miniaturized robust, precise and accurate devices capable of real-time and online analysis and the sensor technology provides a promising and powerful tool for this purpose ${ }^{5}$. The development of a sensor for the heavy metals in the water is very tedious because of their smaller ionic radii and selectivity issues but the molecular imprinting provides a very straight forward methods for the development highly selectivity recognition materials towards the analyte of interest ${ }^{6}$. This study focuses on the development of such a miniaturized ion imprinted polymers and their composites with functionalized graphene based piezoelectric and electrochemical sensors for the detection of heavy metal ions namely; $\mathrm{Cr}^{3+}, \mathrm{Fe}^{3+}, \mathrm{Zn}^{2+}$ and $\mathrm{As}^{5+}$ in water. For the molecular imprinting of heavy metals ions, we followed bulk molecular imprinting methodology while duel electrodes quartz crystal microbalance (QCM) and IDEs were used as transducers. Ion imprinted polymers and their composites (MIPs and functionalized graphene) were prepared with optimized recipes and then their thin films ranging from $120-140 \mathrm{~nm}$ thickness were generated by spin coating onto dual electrodes QCM sheets and IDEs. Sensors were exposed to different concentrations of respective
\end{abstract}

template metal ions and sensors showed substantial sensitivities with limit of detection for $\mathrm{Cr}^{3+} 5 \mathrm{ppb}$ while $6 \mathrm{ppb}$ for $\mathrm{Fe}^{3+}$ sensor, $4 \mathrm{ppb}$ for $\mathrm{Zn}^{2+}$ sensor and $3 \mathrm{ppb}$ for $\mathrm{As}^{5+}$ sensor. The sensitivity of the sensors were enhanced by blending their MIPs with functionalized grephene and the difference can be seen from figure 1 in case of $\mathrm{As}^{5+}$ sensor.

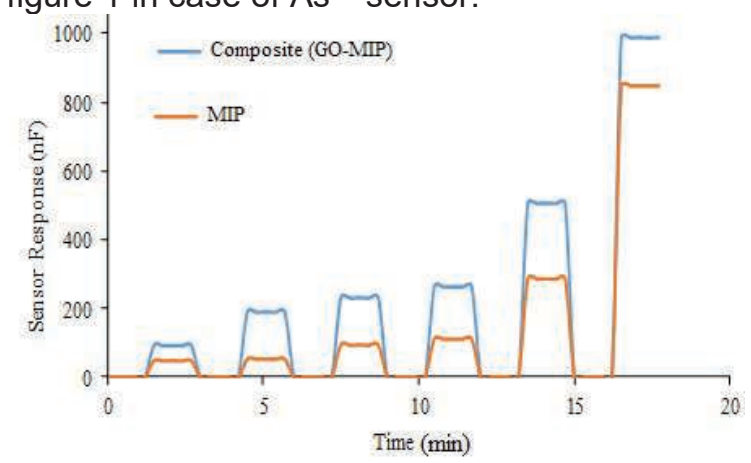

Figure 1 Sensor respose of MIPs and composite towards 5-50 ppb of $\mathrm{As}^{5+}$.

These sensors are highly specific and selective for their respective analyte in the presence of other competing metal ions with quite similar atomic masses, atomic radii and oxidation states as shown by figure 2 in case of arsenic sensor. This substantial sensitivity and selectivity of each sensor make them as promising tools for the online and real-time monitoring of heavy metals in waste, drinking and freshwater sources. 


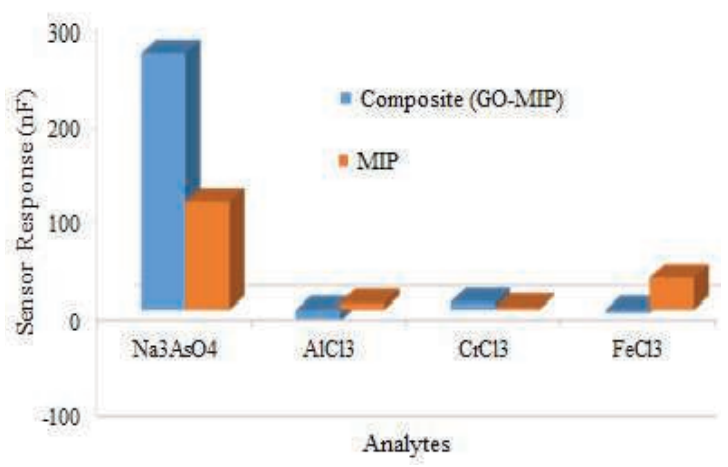

Figure 2 Selectivity behaviour of As sensor towards the various heavy metals at concentration of 30ppb each.

References:

1- Y. Huang, F. Li, C. Ye, M. Qin, W. Ran and Y. Song , Scientific Reports 5, 9724(2015); doi:10.1038/srep09724.

2- N. Iqbal, G. Mustafa, A. Rehman, A. Biedermann, B. Najafi, PA. Lieberzeit, FL. Dickert, Sensors 10,6361-6376(2010); doi:10.3390/s100706361.

3- H. Perry, M. Splendore, A. Chien, NK. Davis and RN. Zare, Angewandte Chemie 50, 250-254(2011);

DOI: 10.1002/anie.201004861.

4- FL. Dickert, PA. Lieberzeit, P. Achatz, C. Palfinger, M. Fassnauer, E. Schmid, W. Werther, G. Horner, Analyst 129:432437(2004); DOI: 10.1039/B315356H.

5- Y. Yang, SK. Seidlits, MM. Adams, VM. Lynch, CE. Schmidt, EV. Anslyn and JB. Shear, Journal of American Chemical Society 132: 13114-13116(2010);

DOI: $10.1021 / j a 1040013$.

6- M. Yasinzai, G. Mustafa*, N. Asghar, I. Ullah, P. A. Lieberzeit, M. Zahid, U. Latif, Journal of Sensors, 1,1-14(2018). 